\title{
EL PROFESOR DE EDUCACIÓN FÍSICA Y LA INTELIGENCIA EMOCIONAL
}

\author{
Margarita Gutierrez Moret \\ Raquel Ibañez Martínez \\ Facultad de Psicología, Magisterio y Ciencias de la Educación. \\ Universidad Católica de Valencia San Vicente Mártir
}

https://doi.org/10.17060/ijodaep.2017.n1.v3.1010

Fecha de Recepción: 8 Enero 2017

Fecha de Admisión: 1 Abril 2017

\section{RESUMEN}

La asignatura de educación física tiene unas características que la hacen idónea para el desarrollo de las habilidades de gestión y regulación emocional en los alumnos. Pero la inteligencia emocional que está desarrollándose en las aulas de educación física debe tener como paso previo la formación de los docentes en estas habilidades emocionales. En este trabajo se presentan los resultados del análisis sobreel perfil emocional de los futuros profesores de educación física a través de una medida de autopercepción y habilidad en Inteligencia Emocional.

Palabras Claves.: Inteligencia emocional, educación emocional, formación, profesores, docentes

\section{ABSTRACT}

The subject of physical education has characteristics that make it suitable for the development of management skills and emotional regulation in students. But the emotional intelligence that is developing in physical education classrooms must have as a previous step the training of teachers in these emotional skills. This paper presents the results of the analysis on the emotional profile of future physical education teachers through a measure of self-perception in Emotional Intelligence.

\section{INTRODUCCIÓN}

La educación emocional, en la última década, ha sido un tema de gran interés desde el ámbito educativo,y desde de la psicología positiva la llamada inteligencia emocional (IE).

Cada vez los profesores y educadores en general están teniendo en consideración las competencias emocionales en su trabajo diario y reconociendo la necesidad de desarrollar la inteligencia emocional de sus estudiantes.

Desde que apareció en la literatura científica el concepto de IE (Salovey y Mayer, 1990) diversos 


\section{EL PROFESOR DE EDUCACIÓN FÍSICA Y LA INTELIGENCIA EMOCIONAL}

modelos han tratado de definirla. Este estudio se basa en uno de los modelos más consolidados, el de Salovey y Mayer, que considera la IE como "la capacidad para percibir, valorar y expresar las emociones con exactitud; la capacidad para acceder y generar sentimientos que faciliten elpensamiento; la capacidad para entender la emoción y el conocimiento emocional; y la capacidad pararegular las emociones y promover el crecimiento emocional e intelectual" (Mayer ySalovey, 1997:10). Desde esta aproximación la IE se refiere a un conjunto de habilidades que permiten a las personas solucionar problemas de manera adaptativa promocionando el crecimiento emocional.

Gran parte de los estudios sobre el tema han tratado de medir la IE y sus implicaciones en el mundo educativo, en su relación con los docentes (Bisquerra, 2005; Bueno, Teruel y Valero, 2005) y los alumnos (Extremera y Fernández Berrocal, 2003; Fernández-Berrocal y Ruiz, 2008). Estos trabajos han contribuido a consolidar la importancia de la IE en el proceso educativo, a pesar de las limitaciones que existen en su conceptualización y medición. Hoy en día también sabemos que la IE se puede trabajar en todas las etapas educativas, desde la educación infantil hasta la educación secundaria.

Pese a que son muchos los estudios que han mostrado los beneficios de la mejora de la IE en el rendimiento académico, el bienestar de los alumnos y el clima de aula (Extremera y FernándezBerrocal, 2003; Fernández-Berrocal y Ruiz, 2008), en el proceso de formación de los profesores de secundaria la IE no ha recibido la atención adecuada (Gutiérrez, Ibáñez y Aguilar, 2014).

La educación emocional supone incorporar la IE en las escuelas, pero esto no solo tienen que ver con las destrezas y el aprendizaje de los alumnos, también debe incorporarse en la formación docente.La inclusión de la educación emocional en la formación del profesorado de secundaria tiene un doble objetivo, uno que va dirigido a la propia profesión docente, a favorecer el desarrollo de las competencias emocionales que pueden ayudar al profesor a un mejor desempeño de su trabajo y a minimizar y prevenir el estrés asociado a las situaciones conflictivas que pueden vivirse en los centros escolares. Al mismo tiempo la formación emocional en el profesorado va dirigida a potenciar el desarrollo global del alumno.Se entiende la educación emocional como una educación para la vida que debe estar presente a lo largo del currículo académico (Bisquerra, 2005).

Los alumnos pasan muchos años de su vida en el sistema educativo, donde el profesor tiene un papel fundamental en el desarrollo de actitudes, comportamientos y emociones. Los profesores son agentes activos en el desarrollo afectivo de sus alumnos, y deberían ser conscientes de sus propias emociones (Bar-On, 2006), e incorporar estas habilidades emocionales en su trabajo (Extremera y Fernández-Berrocal, 2005). Es necesario que los profesores desarrollen una gran capacidad para regular sus propias emociones, para manejar las de sus alumnos, las diferentes situaciones de interacción personal, no solo relacionadas con el proceso de aprendizaje en el aula, sino también en los contactos con padres, compañeros y otros agentes de la comunidad educativa.

La educación física ha sido un área poco abordada desde la perspectiva de la IE, a pesar de que la emoción está estrechamente ligada a las teorías motivaciones y estas últimas sí han generado una gran contribución científica en el área del deporte y la educación física (Cera, Almagro, Conde y Sáenz-López , 2015)

El Ministerio de Educación propone que la finalidad principal de la educación física es desarrollar en las personas su competencia motriz, entendida como la integración delos conocimientos; procedimientos; actitudes; y sentimientos, vinculados fundamentalmente a la conducta motora (MECD, 2014). Esta inclusión de los aspectos actitudinales y emocionales también se recoge en el trabajo de Kirk (2008) sobre los retos de futuro de la educación física actual, en el que destaca el papel de esta materia en desarrollo de conductas prosociales en la infancia y adolescencia.

Los beneficios de la actividad física son bien conocidos y se relacionan con efectos positivos en 
muchas variables del contexto escolar, sobre el autoconcepto físico, la concentración, la memoria, el comportamiento en clase y las relaciones con los compañeros (López, Pérez, Manrique y Monjas, 2016). Además la educación física puede educar los aspectos del desarrollo emocional y de las relaciones interpersonales desde la propia acción motriz; usando el juego para abordar los conflictos sociales (Sáez, Lavega, Lagardera, Costes y Serna, 2014).

La educación física tiene unas características destacables para el desarrollo y adquisición de las competencias socioemocionales. Es singular por los diferentes tipos de aprendizaje que se trabajan ylas metodologías tan diversas, que incluyen juegos, expresión motriz, deportes de grupo, etc. La expresión y vivencias de carácter emocional que se generan en esta asignatura merecen también un interés especial por su contribución al desarrollo de la personalidad de los adolescentes (Bisquerra y Pérez, 2007; Duran, Lavega, Planas, Muñoz y Pubill, 2014)

Los alumnos que cursan educación física en la etapa de la educación secundaria están en un momento crucial de su desarrollo socioemocional. Diversos estudios plantean que programas adecuados de educación física en adolescentes generan experiencias emocionales positivas (Moreno, Murcia y Hernández, 2009)promoviendo estilos de vida activos y saludables. Se trata de un contexto de aprendizaje y una etapa vital idónea para la formación en estrategias de regulación emocional.

La inteligencia emocional que está desarrollándose en las aulas debe tener como paso previo la formación de los docentes en estas habilidades emocionales, por lo que debemos avanzar hacia el diseño de propuestas formativas que favorezcan el desarrollo de la IE de los futuros profesores.

La mayoría de los programas de formación del profesorado no contemplan de manera formal el desarrollo de las competencias emocionales (Gutiérrez, Ibañez y Aguilar, 2004; Palomero 2009, Cabello, Ruiz-Aranda y Fernández-Berrocal, 2009). Con el objetivo puesto en el diseño de un plan de formación docente que incluya la IE, se hace necesario conocer antes las habilidades y percepciones en competencias emocionales de los futuros profesores de educación física.

En este trabajo se presentan los resultados de una primera fase de un estudio más amplio sobre la IE en la formación del profesorado, recogiendo el perfil emocional de los futuros profesores de educación física a través de una medida de autopercepción y habilidad en IE.

\section{MÉTODO}

La evaluación de perfil emocional se realizo a través de los siguientes instrumentos:

1. Cuestionario sociodemográfico breve diseñado ad hoc para la recogida de datos básicos como edad, sexo y estado civil.

2. TMMS-24 El Trait Meta Mood Scale-24 (Fernández-Berrocal, Extremera y Ramos, 2004) es una adaptación del TMMS-48 desarrollado por Mayer y Salovey en 1990. La escala se compone de 24 ítems agrupados en 3 dimensiones,: Atención a los sentimientos, entendida como el grado en que las personas creen prestar atención a sus emociones y sentimientos, Claridad emocional 0 cómo las personas creen percibir sus emociones y Reparación de las emociones, definida como la creencia del sujeto en su capacidad para interrumpir y regular estados emocionales negativos y prolongar los positivos.

3. MSCEIT (Mayer, Salovey, and Caruso Emotional Intelligence Test), Mayer, Salovey, y Caruso (2002). En su adaptación española (Extremera y Fernández-Berrocal, 2002).EI MSCEIT es un instrumento basado en tareas de habilidad constituido por 141 ítems que miden lo bien que la gente realiza tareas y resuelve problemas emocionales en ocho escenarios. Las puntuaciones se dividen en dos áreas (Inteligencia Emocional Experiencial e Inteligencia Emocional Estratégica) y cuatro dimensiones (percibir, usar, entender y gestionar las emociones). 


\section{EL PROFESOR DE EDUCACIÓN FÍSICA Y LA INTELIGENCIA EMOCIONAL}

\section{PARTICIPANTES}

La muestra está compuesta por 101 sujetos, alumnos del Máster Oficial de Profesorado de Enseñanza Secundaria de la Universidad Católica San Vicente Mártir de Valencia y Licenciados y Graduados en Ciencias de la Actividad Física y del Deporte. La mayoría de los entrevistados son hombres (90\%), con edades entre los 21 y los 29 años.

Los participantes realizaron la evaluación con la supervisión de un psicólogo y siguieron este orden: cuestionario sociodemográfico breve, TMMS-24 y MSCEIT. En todo momento se informó a los participantes del propósito del estudio y la voluntariedad de la participación en el mismo, asegurando asimismo el anonimato y la confidencialidad de los datos.

\section{ANALISIS DE LOS DATOS}

Los datos obtenidos fueron grabados y analizados con el software estadístico SPSS 19.0 para PC. Se realizó un análisis descriptivo y una comparación de medias mediante la prueba t de Student para muestras independientes con las variables sexo y grupo de edad.

\section{RESULTADOS}

Los resultados del MSCEIT muestran que el nivel de IE general es de 102,53 (dt: 14,876), de las áreas experiencial $(99,31$, dt:12,020), y estratégica $(108,1, \mathrm{dt}: 10,565)$. De las ramas MSCEIT, Ios resultados son: Percepción emocional 101,19 (dt:13,515, Facilitación emocional 97,07(dt: 9,763),Comprensión emocional 108,02 (dt:10,728) y Manejo emocional 106,14 (dt: 11,707).

Los resultados del TMMS-24 muestran que en percepción emocional, un 30\% de los entrevistados tienen puntuaciones bajas; en comprensión y regulación los resultados son muy cercanos a la media.

Estos datos sugieren que los futuros profesores de educación física tienen un perfil de IE similar a la media de población de su edad.

\section{DISCUSION}

A pesar del reconocimiento en la literatura de la necesidad de trabajar las habilidades emocionales de los futuros profesores y contemplar la formación de competencias emocionales en los programas de formación docente(Corcoran y Tomey ,2012), pocos trabajos sobre intervenciones diseñadas para la mejorar la IE han cuidado los aspectos metodológicos (Pertegal, Castejón-Costa y Martínez, 2011)y en ocasiones parten de diversos modelos teóricos que dificultan la validación de los programas. Antes de diseñar y poner en marcha cualquier intervención en los planes de formación de los profesores se hace necesario conocer bien las percepciones y habilidades de ejecución en estas competencias emocionales. Este trabajo es un primer paso para el diseño de una propuesta de modificación de los planes de estudio de formación de profesores de Educación Física que introduzca las enseñanza de habilidades relacionadas con la inteligencia emocional.

Los resultados presentados muestran un perfil de Inteligencia Emocional percibida y de habilidades de ejecución en futuros profesores de Educación Física similar a los jóvenes de su edad. En investigaciones realizadas en otros colectivos sobre IE (Schutte y Malouff, 2012), se constata que no existen diferencias relevantes respecto a la media en población general. Sin embargo, en determinados colectivos profesionales se requiere una mayor capacidad de gestión de las emociones debido a las características propias de su desempeño laboral.

Estos datos son relevantes en cuanto a qué podemos considerar que los profesores deben tener un perfil de IE mayor para hacer frente a las exigencias emocionales de su trabajo docente. Estudios previos han puesto de manifiesto que la IE asume un papel relevante cuando el trabajo implica una demanda emocional intensa (Chernisy Goleman 2001) tal y como ocurre en profesiones como la docencia. 
Conforme a Brackett y Caruso (2007), las habilidades relacionadas con el manejo de las emociones afectan, entre otros, a los procesos de aprendizaje, a la salud mental y física, a la calidad de las relaciones personales y sociales y al rendimiento académico y laboral, razón por la que los profesores debería mejorar los niveles de IE de forma significativa garantizando de esta forma el máximo potencial en su desempeño profesional.

Reconocido el papel de las habilidades emocionales en la educación física, los estudios deben dirigirse hacia la intervención educativa. El diseño de programas y metodologías en la que faciliten el aprendizaje de las competencias emocionales, y la evaluación de los mismos, la gran ausente en la literatura sobre el tema. Se trata de incluir la IE en la formación de los profesores de educación física.La implicación en procesos de formación permanente de los profesores guarda una fuerte relación con la mejora de la práctica profesional en profesores de educación física (Barba, GonzálezCalvo y Barba-Martín, 2014).Las destrezas y conocimientos emocionales son fundamentales para los profesores que deben tomar decisiones efectivas sobre contenido, currículo y pedagogía además de gestionar las dinámicas de clase.

Se propone diseñar un programa de formación específico en IE para los estudiantes del Máster de Formación del Profesorado en la especialidad de Educación Física que refuerce el aprendizaje de las habilidades de percepción, comprensión y regulación emocional.

\section{REFERENCIAS}

Barba, J.J., González-Calvo, G. y Barba-Martín, R.A. (2014). El uso de los diarios del profesorado como instrumento de reflexión sobre la acción. Revista Española de Educación Física y Deportes, 405, 55-63.

Bar-OnRaven (2006). The Bar-On model of emotional-social intelligence (ESI).Psicothema. Vol. 18, supl., pp. 13-25

Bisquerra, R. (2005). La Educación emocional en la formación del profesorado, en Revista interuniversitaria de formación del profesorado, 19 (3), 95-114.

Bisquerra, R., y Pérez, N. (2007). Las competencias emocionales, en Educación XXI, 10, 61-82.

Brackett, M.A. y Caruso, D.R. (2007).Emotionally literacy for educators.Cary, NC:

Bueno, C., Teruel, MP., Valero, A. (2005). La inteligencia emocional en alumnos de Magisterio: la percepción y comprensión de los sentimientos y als emociones enRevista interuniversitaria de Formación del Profesorado, 19 (3), 169-194.

Cabello, R., Ruiz-Aranda, D., y Fernández-Berrocal, P. (2010). Docentes emocionalmente inteligentes, en REIFOP, 13 (1). (Disponible en http://www.aufop.com).

Cera, E.; Almagro, B. J.; Conde, C., y Sáenz-López, P. (2015). Inteligencia emocional y motivación en educación física en secundaria. Retos: Nuevas tendencias en Educación Física, Deporte y Recreación, 27, 8-13.

Chernis and Goleman 2001; Cherniss, C. (2001).Emotional intelligence and organizational effectiveness. In C. Chernissy D. Goleman (Eds.), The emotionally intelligence workplace: How to select for, measure, and improve emotional intelligence in individuals, groups, and organizations (pp. 3-12). San Francisco: Jossey-Bass.

Corcoran, R.P., y Tormey, R. (2012a). Assessing emotional intelligence and its impact in caring professions: the value of a mixed methods approach in emotional intelligence work with teachers. In A. Di Fabio (Ed.), Emotional intelligence: new perspectives and application (pp. 215-238). Rijeka: InTech.

Extremera, N. y Fernández-Berrocal, P. (2003). La inteligencia emocional en el contexto educativo: hallazgos científicos de sus efectos en el aula, en Revista de Educación, 332, 97-116. 


\section{EL PROFESOR DE EDUCACIÓN FÍSICA Y LA INTELIGENCIA EMOCIONAL}

Extremera, N. y Fernández-Berrocal, P. (2003). La inteligencia emocional en el contexto educativo: hallazgos científicos de sus efectos en el aula. Revista de Educación, 332, 97-116.

Extremera, N., y Fernández-Berrocal, P. (2005). Inteligencia emocional percibida y diferencias individuales en el meta-conocimiento de los estados emocionales: una revisión de los estudios con el TMMS, en Ansiedad y Estrés, 11(2-3), 101-122.

Extremera, N., y Fernández-Berrocal, P. (2005). Inteligencia emocional percibida y diferencias individuales en el meta-conocimiento de los estados emocionales: una revisión de los estudios con el TMMS. Ansiedad y Estrés, 11(2-3), 101-122.

Fernandez-Berrocal, P., y Ruiz-Aranda, D. (2008). La Inteligencia emocional en la Educación. Revista Electrónica de Investigación Psicoeducativa. 15,Vol 6 (2), 421 - 436.

Fernandez-Berrocal, P., y Ruiz-Aranda, D. (2008). La Inteligencia emocional en la Educación, en Revista Electrónica de Investigación Psicoeducativa. 15,Vol 6 (2), 421 - 436.

Gutiérrez, M, Ibáñez, R, y Aguilar, R. (2013) Apuesta por la formación de competencias desde la inteligencia emocional. Reflexiones sobre su importancia en la docencia, en Revista Edetania: estudios y propuestas socio-educativas, (44), 77-92

Kirk, D. (2008). Los futuros de la Educación Física: la importancia de la cultura física y de la «idea de la idea» de Educación Física. En AA. VV. Actas V Congreso Asociación Española de Ciencias del Deporte. León: Universidad de León. (CD-R).

López, Pérez, Manrique y Monjas, (2016) Los retos de la Educación Física en el Siglo XXI Challenges of PhysicalEducation in XXI Century Retos, número 29, 2016

Mayer, J. D., y Salovey, P. (1997). What is emotional intelligence? En P. Salovey y D. Sluyter (Eds), Emotional Development and Emotional Intelligence: Implications for Educators (pp. 3-31). Nueva York: Basic Books.

Palomero, P. (2009). Desarrollo de la competencia social y emocional del profesorado: una aproximación desde la psicología humanista, en REIFOP, 12 (2), 145-153.

Parlebas, P. (2001). Juegos, deporte y sociedad. Léxico de praxiología motriz. Barcelona: Paidotribo.

Pertegal, M.L., Castejón-Costa, J., Martínez, M.A. (2011). Competencias socioemocionales en el desarrollo profesional del maestro, enEducación XXI, 14.2, 237-260.

Real Decreto 126/2014, de 28 de febrero, por el que se establece el currículo básico de la Educación Primaria (BOE, no 52 de 1 de marzo de 2014; 19.349-19.420).

Sáez de Ocáriz; Lavega, Lagardera, Costes y Serna (2014). ¿Por qué te peleas? Conflictosmotores y emocionesnegativas en la clase deEducación Física: el caso de los juegos de oposición Educatio Siglo XXI, Vol. 32 no 1 - 2014, pp. 71-90

Salovey, P. y Mayer, J. D. (1990). Emotional intelligence. Imagination, Cognition, and Personality, 9 (3), 185-211.

Schutte, N.yMalouff, J.M. (2012), “Priming ability emotional intelligence”, Intelligence, 40, 614-621. SEL media. 